\title{
Wind tunnel for studies of latent heat storage
}

\author{
Václav Tesar̆ ${ }^{1, a}$ \\ ${ }^{1}$ Institute of Thermomechanics v.v.i., Czech Academy of Sciences, Dolejškova 5, 18200 Praha-Kobylisy, Czech Republic
}

\begin{abstract}
When a heated solid body temperature reaches the melting point, temperature stops increasing and remains constant until the whole body is completely molten. The heat input during this melting is spent on freeing the body molecules. This latent heat of melting remains inside the body and may be released when the body is cooled and solidifies. This heat was suggested, already several decades ago, for storing thermal energy. The advantage it offers is avoiding high temperature differences - which otherwise decrease effectiveness of storage (by inevitable heat escape by conduction). Also the mass of the body needed to store a given amount of heat is much smaller. For investigations of the melting and solidification processes a special wind tunnel has been designed and is being built in this study. The tested sample of phase change material, encapsulated in a spherical shell, will be exposed in the tunnel to recirculating hot air flow in a $140 \mathrm{~mm}$ x $140 \mathrm{~mm}$ test section. Sudden decrease in air flow temperature is made by shifting away the whole closed-circuit part of the tunnel and exposing the test section to flow of cold (room temperature) air.
\end{abstract}

\section{Introduction}

One of the main problems with energy - perhaps the crucial one - is mismatch between availability and demand. This creates a strong interest in efficient energy storage. Of the existing principles listed in Fig. 1, this paper is concerned with temporary storing of heat between the times when it is abundant and when demanded. While some other storage principles need a mechanical machinery or another complex system, the heating and cooling of a body is extremely simple and fail-safe. Intensity of the heating and cooling processes in the simplest case depends on available temperature differences - while total storage capacity depends on the mass of the charged body. High temperature differences suffice with a small storage body but may cause problems - such as too much heat escaping through inevitably imperfect insulation by conduction.

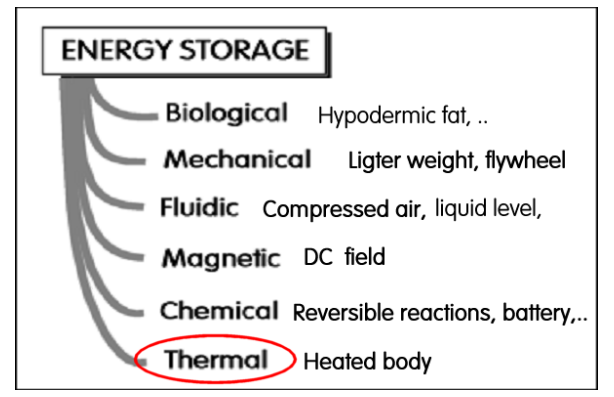

Fig. 1. Among energy storage methods, the thermal storage handles lower quality energy, not directly convertible into other forms - particularly not into the most often demanded electric energy. It is thus particularly useful for simple applications such as keeping a more or less constant temperature of living bodies (humans) and objects with which humans come in contact.
An interesting solution avoiding the high temperatures and yet needing only rather small heated body is offered by using phase changes. In principle, the charging and discharging can take place in the region of constant temperature seen on the equilibrium states diagram (valid for paraffins) in Fig. 2.

The variability of parameters of a substance is governed by the Gibb's Phase Rule [1]

$$
\mathbf{F}=\mathbf{3}-\mathbf{P}(1)
$$

- where $\mathbf{F}$ is the number of degrees of freedom while $\mathbf{P}$ is the number of phases in mutual contact. With a singlephase material considered here it is possible to vary pressure and temperature independently $(F=2)$. Upon reaching the phase change surface (Fig. 2) the degrees of freedom reduce to $F=1$. This means the temperature of an isobarically heated material remains constant until all the investigated contents is changed from the state $\mathbf{a}$ into b. The heat input without corresponding temperature increase is described as "latent heat", the term introduced in 1762 by J. Black, [2] (professor of "Medicine and Chemistry" at the University of Edinburgh) in experiments supported by Scottish whisky manufacturers.

In principle it is possible to use for the storage the melting as well as vapourisation phase changes. It was the latter that was studied by Black - but practical aspects in the storage problem generally give preference to the former. Magnitudes of the stored heat is presented in Fig. 3 . The same amount of heat is released from the melt during its solidification. The advantages obtainable from the (very nearly - note the differences needed at the process beginning and end) isothermal energy storage were soon recognised as attractive possibility - apparent by comparing in Fig. 3 the difference between the final state $2 *$ without the phase change and 2 with the latent heat. Attractiveness of the latent heat storage idea is reflected in the large and steady rising numbers of annually published research papers on this subject, 


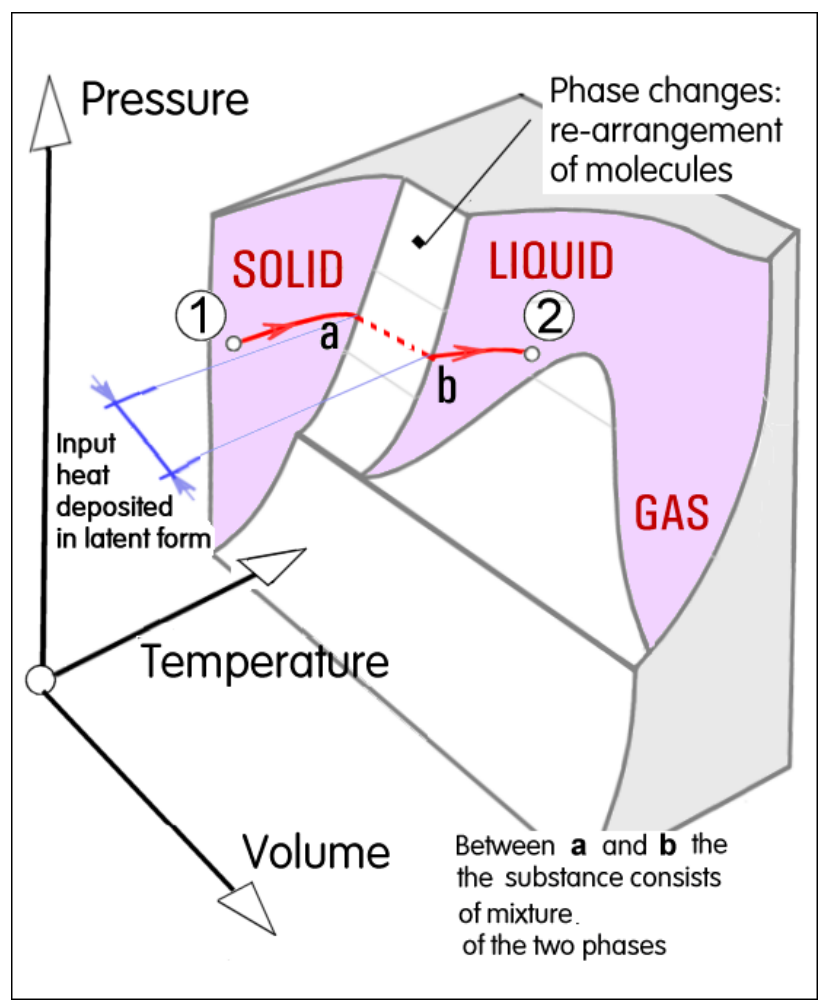

Fig. 2. When a solid substance is heated, the limit $\mathbf{a}$ is reached at which all additional thermal input is used to liberate the molecules into free motion in liquid phase. Temperature is not increased until all molecules undergo the phase change to $\mathbf{b}$. The diagram applies to paraffins the volume of which increases during melting (solid paraffin body does not float on the liquid phase surface and sinks to the bottom).

presented in Fig. 4. Many storage processes among those listed in Fig. 1 are proposed and currently developed aiming at the problem of mismatch in electric energy, nowadays increasing in importance as the result of poor controllability of nuclear reactors on one hand and unreliability of renewable energy sources on the other one. Thermal energy storage, however, is not really suited for this particular purpose. Converting heat into electricity at industrial scale requires engines operating in a thermal cycle to evade the fact that thermal energy -

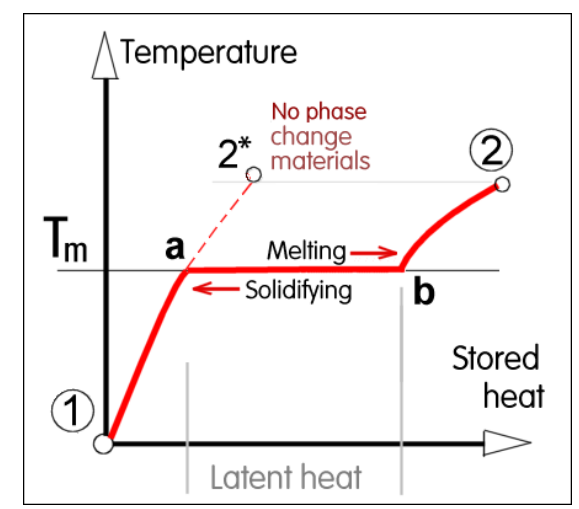

Fig. 3. Dependence between variations of temperature and internal thermal energy of a substance with and without phase change between the input state 1 and final states 2 or $2 *$. energy of chaotic motions - is of lower "quality" that the organised motion of electrons in electric conductors.

Nevertheless, there are many cases where the electric form is not necessary. There are energy consumers needing it in the thermal form. In particular, there is a significant activity in development of "smart" thermal insulators accumulating heat for a certain, usually not long period at quite low temperature levels. Especially promising is the current development of "smart" textiles, containing tiny encapsulated volumes of the phase change material among the fibres of the woven yarn. With this textile material, typically for use in battle-dress uniforms in desert combat areas, the latent-heat beads can cool the soldier during the hot desert day (while being charged by the solar heat) - and heating him in the cold desert night (discharging the accumulated heat) [3]. Another very typical application is cooling the heat generated in very compact, high-density electronic components. In particular, there are now active research projects investigating cooling of hand-held smart phones [4] that in the most compact versions can become heated to temperature levels above $45{ }^{\circ} \mathrm{C}$ - the limit at which plastic objects in experiments [5] could be held for a long time in hand.

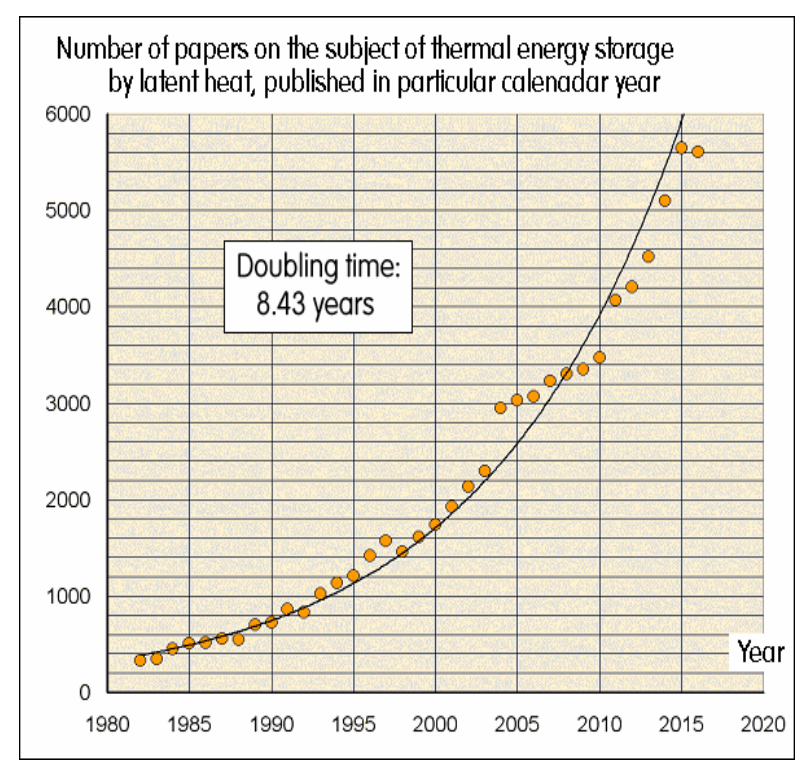

Fig. 4. Interest in storage of thermal energy in latent heat of a phase change has been steadily increasing as documented by the annual number of published papers - data from Scopus.

\section{Phase change materials}

The earliest observations of the processes associated with melting and solidification phase changes used to involve water and its change into ice. These, however, take place at inconveniently low temperature from the point of above mentioned human-related applications. Interest therefore shifted to studies of other materials, especially those that could be used in the temperature levels typical for living bodies - indicated by the horizontal red line in Fig. 5. The material developments resulted in four substance families 


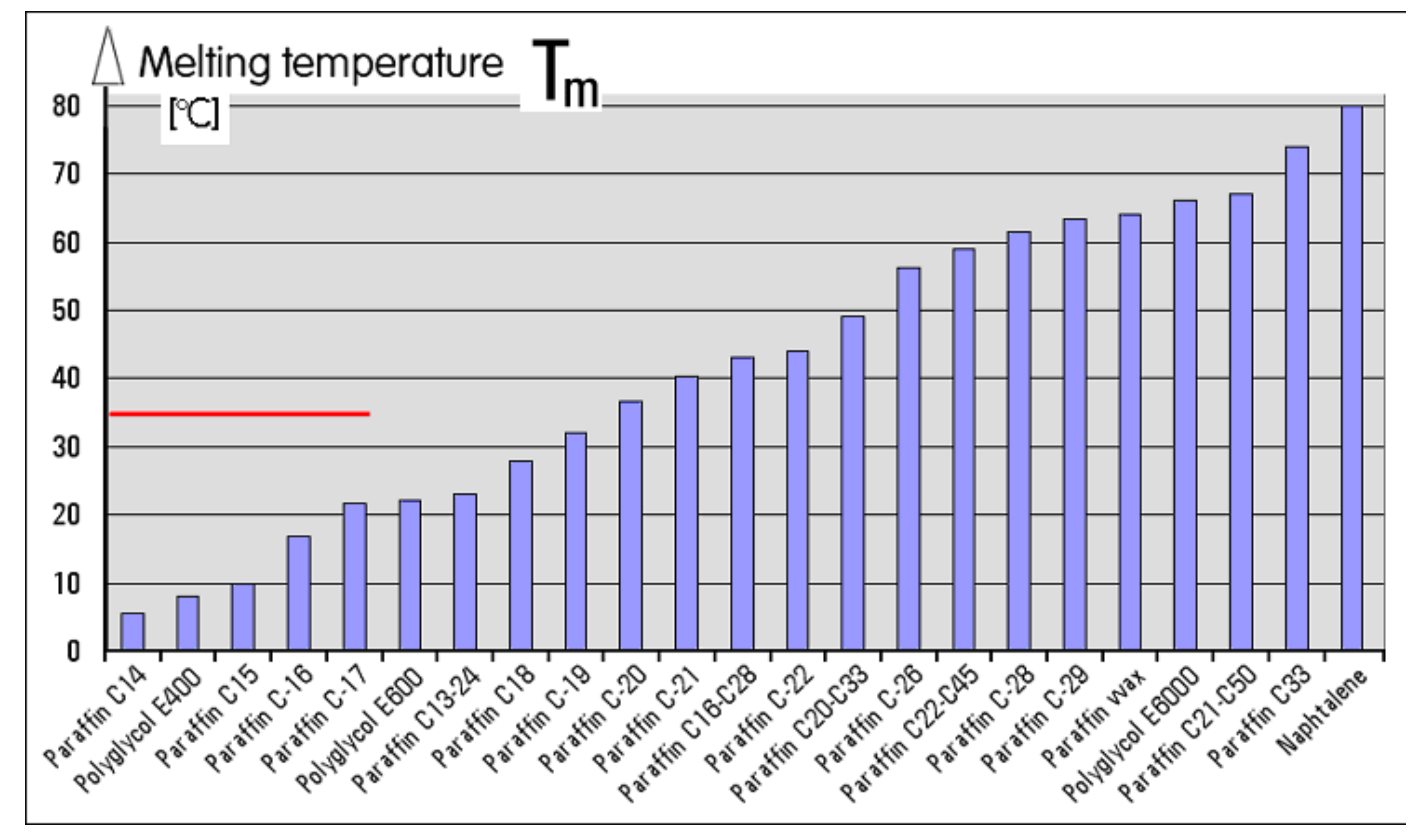

Fig. 5. Present prevailing development interests in application of latent heat storage tend to concentrate on keeping comfortable thermal conditions for humans (red line) and also of devices and instruments that should be comfortably touched even if they generate heat. As shown here, existing range of commercially available paraffins offers a suitable choice of the melting temperature level.

considered to be most promising - not only from the point of view of the melting temperatures, but considering also several other criteria. The total list of requirements is as follows:

(1) Suitable level of the melting temperature $\mathrm{T}_{\mathrm{m}}$

(2) Large enthalpy of phase change $\mathrm{e}_{\mathrm{L}}$ (Fig. 8)

(3) High thermal conductivity

(4) Little or no superheating and subcooling

(5) Availability

(6) Low cost

(7) Inflammable as well as chemically inert

(8) Non-toxic and convenient for manual handling

The importance of the proper melting temperature has finally led to present-day commercial availability of phase change materials with quite finely adjustable $T_{m}$ values, an example of which is presented in Fig. 5. This now provides an opportunity to concentrate on other properties from the above list.

The importance of large enthalpy of fusion - i.e. the horizontal width $e_{L}$ of the phase change zone between a and $\mathbf{b}$ in Figs. 3 and 8 is also obvious. Typical values for the four material families are presented in Fig. 6 .

It may be perhaps less apparent why it is also important for the phase change material to have high thermal conductivity - both in the solid and liquid phases. The lower is the heat flow resistance, the smaller are the deviations from temperature constancies at both ends of the heating curve shown in Figs. 3 and 8, i.e. the difference between the points $\mathbf{1}$ and $\mathbf{a}$ in the discharging (solidification) regime and between the points $\mathbf{b}$ and $\mathbf{2}$ in the charging (melting) process.

Some materials exhibiting a proper melting temperature value $T_{m}$ and thus seemingly suitable for the thermal energy storage may be actually not practically useful for this role because of their large hysteresis of the heating curve. Instead of the single horizontal line between $\mathbf{a}$ and $\mathbf{b}$ in Figs. 3, 7 and 8, there may be two horizontal lines at different temperatures - one for heating and the other for cooling. The subcooling means the molten phase does not solidify at the nominal $\mathrm{T}_{\mathrm{m}}$ - and the superheating means the material stays solid at temperatures above the nominal $\mathrm{T}_{\mathrm{m}}$ value. The four families of materials most promising for meeting the criteria (1) to (8) in the previous page are:

(a) Low melting temperature metals,

(b) Paraffins

(c) Linear alkyl hydrocarbons

(d) Salt hydrates

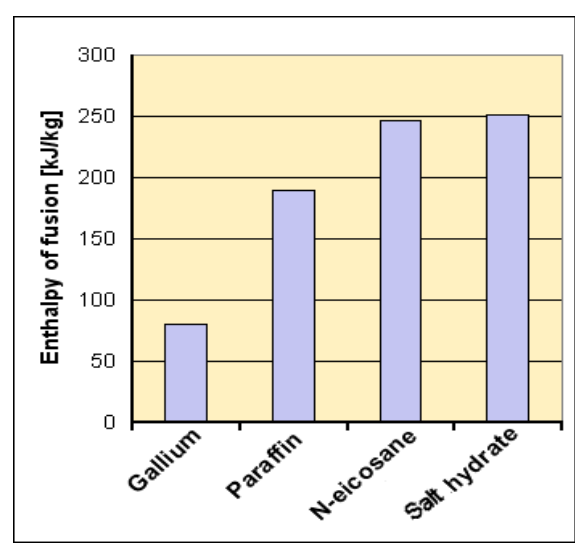

Fig. 6. Selected examples of heat stored per unit of mass - one from each of the four discussed latent-heat storage material families: low-melting-point metals, paraffins, alkyls, and salt hydrates. Of course, high value of the fusion heat $e_{L}$ shown here is, together with the temperature presented in Fig. 5, the prime factor in choice of the used material. There are, however, other properties that may be of importance in particular cases. 


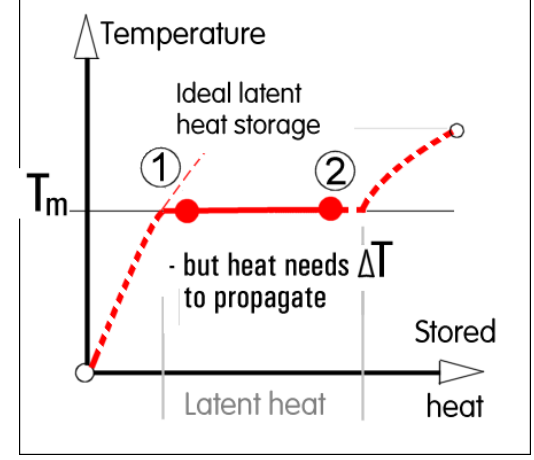

Fig. 7. Ideal loss-less heat storage would follow the constant temperature level as shown here between $\mathbf{1}$ and $\mathbf{2}$. Unfortunately, transport of heat to or from the storage body is not possible without temperature differences necessary - Fig. 8 - for charging as well as discharging.

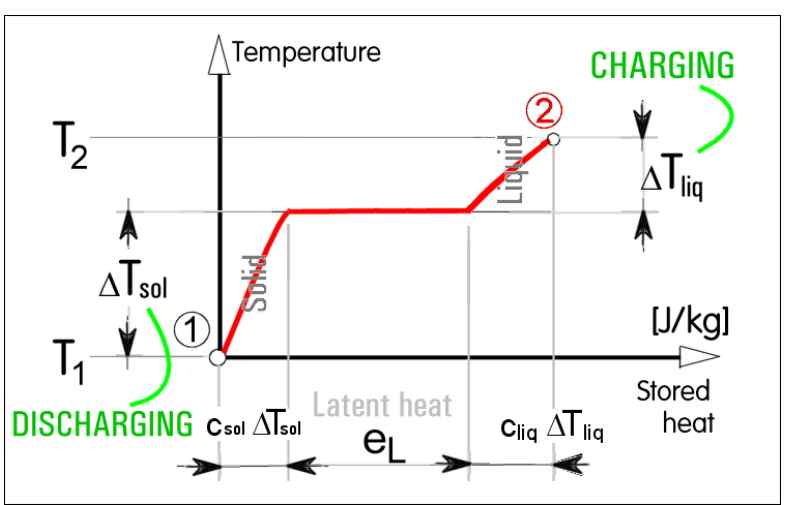

Fig. 8. Actual latent-heat storage contains the inevitable conductivity-governed transport into or from the storage material. The inevitable conduction parts of charging and discharging, between $\mathbf{1}$ and $\mathrm{T}_{\mathrm{m}}$ as well as between $\mathrm{T}_{\mathrm{m}}$ and $\mathbf{2}$, decrease storage efficiency.

Among the metals remaining liquid at low temperatures, the most attractive from the here discussed point of view seems to be gallium, melting nominally at $\mathrm{T}_{\mathrm{m}}=29,76^{\circ} \mathrm{C}$. The temperature $\mathrm{T}_{\mathrm{m}}$ may be adjusted to a desirable value by alloying gallium with other, higher melting point metals. Advantage of gallium is its availability (it is in production at quite large scale for use in the plutonium-gallium alloy in nuclear weapons, e.g. [16]).

Gallium latent heat of melting is $80.3 \mathrm{~kJ} / \mathrm{kg}$, Fig. 6 . This is not much, but would be acceptable if there were not other problems. One of them is quite large subcooling $[6,7]$. Also unpleasant is the fact that gallium attacks most metals by diffusing into their lattice thus making the other metal brittle. Many low-melting-point metals, such as caesium and rubidium are even less suitable, being highly reactive and toxic. Commercially available is the alloy called "Cerrolow", with melting temperature $\mathrm{T}_{\mathrm{m}}=$ $47.2{ }^{\circ} \mathrm{C}$. Another commercially available alloy is the sixcomponent eutectic consisting of Bi-Pb-Sn-Cd-In-Tl exhibiting $\mathrm{Tm}=41.5^{\circ} \mathrm{C}$.

Organic substances considered or used in the phasechange material heat storage role due to having reasonably low melting temperature $T_{m}$ are of two types.

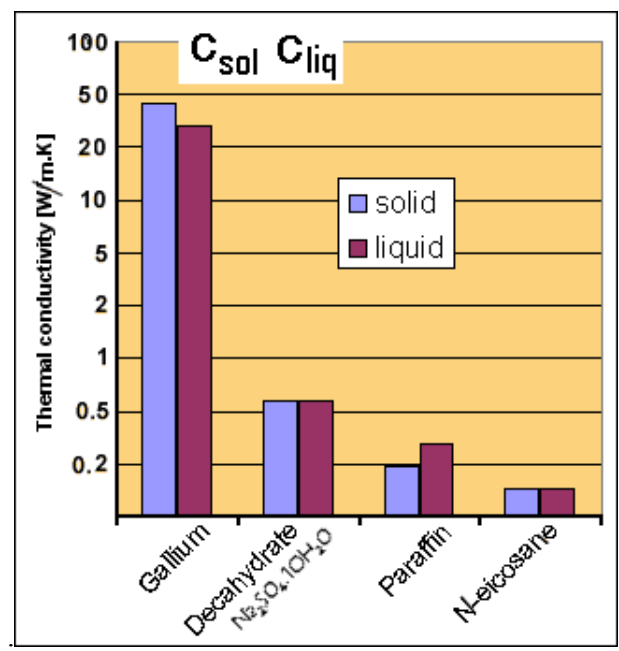

Fig. 9. Characteristic property of the discussed four families of phase change materials are their very different thermal conductivities - note that the values are here plotted using logarithmic scale. The different conductivity coefficients can significantly influence the overall efficiency of the storage.

One of them are paraffins with various $T_{m}$ levels listed in Fig. 5. Their latent heat of fusion (Fig. 6) is higher than that of liquid metals, and their other advantages is the fact that they are chemically inert and friendly in manual handling. They are also commercially available at low cost. Their weak point is typically very low thermal conductivity, cf. Fig. 9, and - perhaps less importantly - also their mild flammability.

Paraffins are at present attractive objects for experimental investigations of latent heat - and are currently often discussed in heat storage literature - due to the useful fact that their liquid and solid phases are easily discriminated visually: molten paraffins are transparent and allow observation of the decreasing or increasing volume of the solid phase, which is opaque.

Other family of interesting organic compounds are alkyls - linear hydrocarbons. Typical, quite widely considered because of some excellent properties, is particularly N-eicosane - listed in Figs. 6 and 9. Alkyls in general are chemically stable, non-corrosive, exhibit no subcooling and posses very high latent heat per unit of weight - Fig. 6 . On the negative side they are flammable (which is not so important property when used inside an encapsulation) and possess very low thermal conductivity, as is presented in Fig. 9 (note the logarithmic scale).

The fourth family of materials currently considered are salt hydrates, inorganic compounds. They were and still are objects of several investigations because of their absolutely highest known values of enthalpy of fusion, cf. Fig. 6. In particular considered as quite attractive is sodium sulfate decahydrate $\mathrm{Na}_{2} \mathrm{SO}_{4} \cdot 10 \mathrm{H}_{2} \mathrm{O}$. Its problem is separation effect in solidification: the solid salt generated in the discharging process settles down at the bottom of the vessel in which this material is kept. As a result of this spatial differentiation the salt ceases to be easily available for recombination with water, necessary during the subsequent charging part of the cycle.

The question of low thermal conductivity, which is a typical property of the otherwise quite attractive organic 
heat storage materials, is not in general given the adequate attention it deserves. It is not uncommon that comparisons of the effectiveness of alternative processes in literature is evaluated based on the assumption presented in Fig. 7 - that both beginning 1 and end $\mathbf{2}$

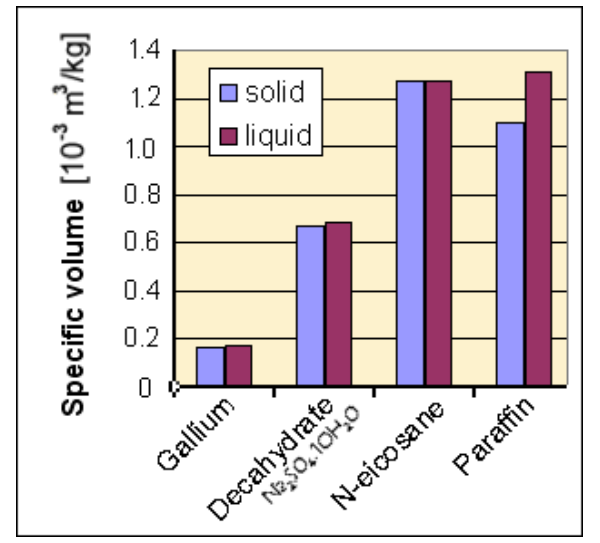

Fig. 10. Also of considerable importance in designing the latent heat storage system are the specific volumes of the materials and the changes that the volume undergoes in the melting and solidification. The typical larger value of the liquid phase means the solid body, instead of floating (like ice) on the liquid surface, would be kept at the bottom of the storage vessel. Values in the diagram are valid for $\mathrm{T} \sim 20^{\circ} \mathrm{C}$.

of the heating process are at their common isotherm. There are actually two facts that should be kept in mind. The first of them is the unavoidable presence of the conduction for charging and discharging, at the ends of the line as shown in Fig. 8. The inlet and outlet temperatures must be above or below the $T_{m}$ isotherm. With no temperature differences the heat would not be transported so that the storage would not work at all. The second fact are wide differences between the conductivity values in the four material families, as seen in Fig. 9 (less apparent there because of the logarithmic scale). The problem is obviously more serious with the organic materials.

There is yet another property of the phase change materials that may play a non-negligible role. It is their specific volume and its variation during the phase change. Of particularly complicating influence may be the large change of volume in the case of paraffins (note the larger difference of the values between the solid and liquid phase in Fig. 10).

\section{Encapsulation}

This volume difference may be so large that the storage material may significantly stress the walls of the vessel in which it is encapsulated - and perhaps even cause them to buckle or collapse. To eliminate this danger, the top of the storage vessel may be left open into atmosphere but then it is necessary to take into account the existence of a quite large part of the capsule occupied in solidification uselessly by the air in the cavity. Perhaps more importantly, the storage system must ensure that the opening for the communication of the vessel with the atmosphere stays always on capsule or vessel top.
Keeping the hole at the required positioning is not so easy task as it might appear.

For his decision on the overall layout of thermal energy storage system with phase change materials, the designer must consider the alternative configurations ensuring that the material in its liquid phase remains where he want it to be - and not spilled elsewhere. This means there has to be for this purpose an encapsulating vessel or cavity.

As shown in Fig. 11, there are three available basic configurations used for containing the phase changing material and keeping it immobilised:

(a) Storage in a tank - with external or internal heaters

(b) Encapsulation - the material is kept in many smaller capsules to decrease the heat transport distance

(c) Microencapsulation: large number of very fine capsules, usually of size less than $1 \mathrm{~mm}$

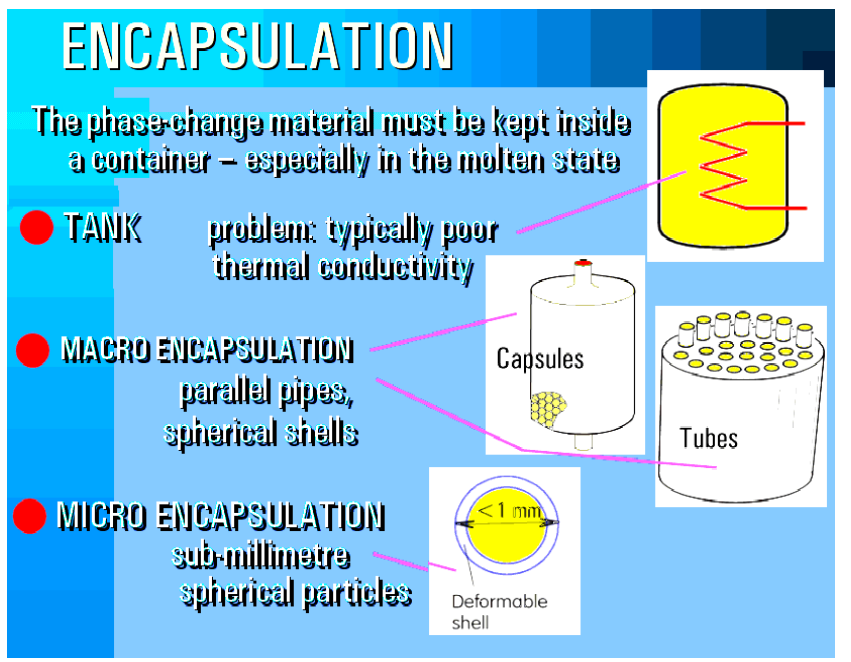

Fig. 11. Especially during the melting process - and, of course, also later when the phase changing material is in the molten state - it is necessary to keep this material within a suitable container preventing the escape. There are three basic configurations.

(a) Tank (or a large cavity inside the solid body): It is the simplest and also least expensive approach. The total volume of the phase change material is kept inside a single space (or perhaps in a few smaller-size spaces). Transfer of heat into the molten material in this case takes place across a quite large internal distances. This may be suitable for slowly operating storage systems, with time scales typically of the order of hours. It may be nevertheless necessary to select the phase change material exhibiting a high thermal conductivity (Fig. 9). The thermal transport may be influenced by the natural convection in the molten material - the convection intensity may be significant due to the larger size of the tank. The heat transport distances may be decreased (thus decreasing the time scale) by complex shaping of the heater, perhaps providing it with some fins or ribs. 


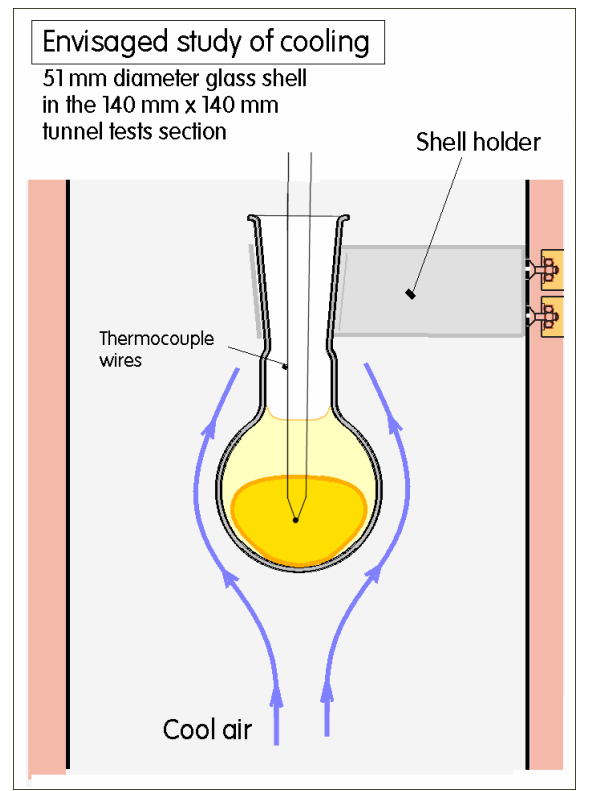

Fig. 12. Proposed configuration of the planned experiments. The heating/cooling fluid (air) flows vertically past the scaledup model of microcapsule. The spherical shell is actually a commercially available glass vessel (flask) attached to the holder at the vessel neck.

(b) Storage material encapsulated: In this case the total volume of the phase change material is divided into a considerable number - or even large number - of solid containment shells. The disadvantage of this approach is increased size and, more important, higher cost of the storage system. On the other hand, the encapsulation improves storage dynamics by decreasing significantly the distance over which the heat is transported. Typical shell configurations are pipes or spheres, Fig. 11. It is also necessary to provide a suitable mechanism for carrying the heat up to each shell. Typical solution is use of another, auxiliary heat carrying liquid which fills the space between the shells on their outer side.

(c) Microencapsulation. Essentially similar to (b) but with substantially smaller shells, typically with their outer size less than $1 \mathrm{~mm}$. This can decrease even more the response time of heating or cooling. Of course, making the large number of the small shells as separate objects would be laborious and therefore expensive. This is circumvented by the shells grown or deposited on the surface of a phase change material droplets - usually by a chemical (or physical) deposition process. Typically of spherical shape, the shell material must be deformable to account for the volume changes during the phase transition. The microencapsulation is a rather new idea now increasing in popularity - especially for the abovementioned use in the "smart" textiles where the submillimetre capsules are fixed between the textile fibres.

\section{Planned experiments}

Because of the expected later use of the information gained in experiments for applications similar to the thermal-insulation hot climate field service uniforms, planned configuration in the tests is with the phase

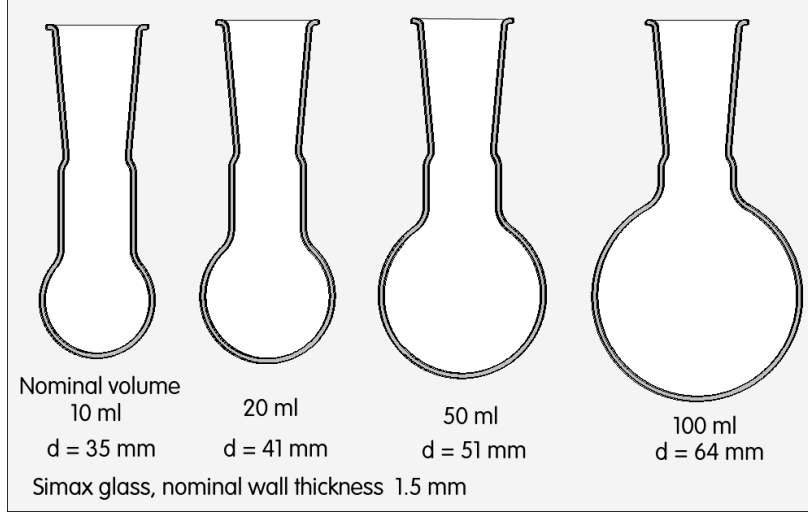

Fig. 13. Vessels obtainable from the supplier to be used in the test runs as the scaled-up shell model. The four available sizes can be usefully applied for investigation of Reynolds number effects at the same air flow velocity. The inlet necks on the top are, of course, a deviation from the ideal spherical geometry, but the downstream location is hopefully in the wake of the flow.

change material filling an internal space of spherical shells. Since the parameter characterising the progress of the phase change processes is going to be the volume fraction of the material in its solid phase, the experiments are planned to use paraffins - due to their transparency in molten state. The fraction will be computed from timed camera-record images of the boundaries of visibly not yet molten solid-state material (as it is approximately shown near the shell bottom in Fig. 12). Precision of the evaluated boundaries is, of course, the better the larger is the investigated object. This is the reason for selecting for observation and recording only a single microcapsule body model, scaled up substantially.

The tests are going to be set up as shown in Fig. 12, with the spherical shell containing the paraffin exposed to flow of air that may be alternatively hot (for melting) or cold (for studies of solidification). The scaling up will also make possible placing into various location inside the paraffin tiny thermocouple probes for local temperature measurement. Of course, also the temperature of the air flowing past the capsule model will be measured by thermocouples, mainly for computing from the air temperature its viscosity. This is necessary for identification of the flow Reynolds number.

$$
\mathrm{Re}=\frac{\mathrm{Wd}}{\nu}
$$

- where $\mathrm{w}[\mathrm{m} / \mathrm{s}]$ is the characteristic velocity of the air flow, $\mathrm{d}[\mathrm{m}]$ is the characteristic length, which in the flows past spherical objects is the sphere diameter (Fig. 13), and $v\left[\mathrm{~m}^{2} / \mathrm{s}\right]$ is air viscosity (dependent on temperature). Because of the kinematic viscosity increasing with air temperature and the expected flow velocities being not large, Reynolds number of the flow past the test model are likely to be very low, corresponding to the not very high flow velocities in the microcapsules applications. The walls of the vertical air flow channels will be provided with transparent windows for illumination of the scene and for the lens of externally positioned camera. Spherical shells of constant wall thickness would be difficult to manufacture in the workshop of the Institute of thermomechanics, author's employer, but 
fortunately suitable objects for use in test models were found in small glass flasks - as shown in Fig. 13 manufactured and offered for sale by local supplier of laboratory glass products. On their offer are four flask diameters (all with the same glass wall thicknesses). These size alternatives with geometric similarity make possible testing the transformation of the phase-change variables and equation into the similarity-transformed coordinates. Certain disadvantage of the use of the flasks is the presence of the neck, which the manufacturer makes on the flask top for insertion into it the tested materials. Of course, some access into the internal volume is necessary anyway, but here the necks appear in relation to the flask body diameters somewhat too large, as may be judged by inspection of Fig. 13. It need not be an unsurmountable problem. Wind tunnel aerodynamic experiments with spherical objects, especially at low Reynolds numbers, indicate that the boundary layer on object surface separates and forms downstream from it a wake inside which presence of material objects like the flask neck does not necessarily substantially influence the flow. The wake is filled with more or less chaotic and fast varying vortices. At the extremely low expected Reynolds numbers, from $\operatorname{Re} \sim 10$ to $\operatorname{Re} \sim 100$, there is a certain regularity of the flow with stationary vortex ring inside the wake, which may interact with the flask neck, but preliminary estimates indicate higher Re values.

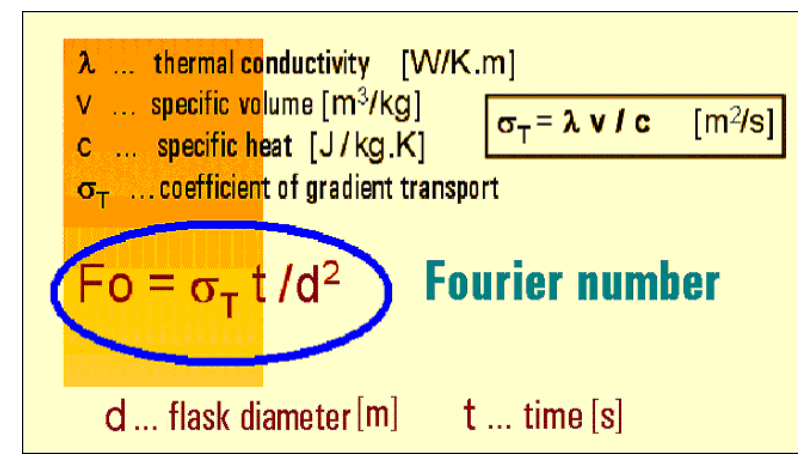

Fig. 14. Fourier number $F_{0}$ is the governing parameter of unsteady heat transfer processes. It may be expected quite safely that in the discussed case it will be the dimensionless measure of melting and solidification time.

The wake past a sphere begins at the location where the boundary layer separates from the body surface. In laminar flows the typical angular distance from the front stagnation point to the separation point is $\sim 80 \mathrm{deg}-$ i.e. already upstream the position where, at $\sim 90 \mathrm{deg}$, the sphere has its largest diameter. It is unlikely, especially in the hot air flow, that the conditions in the here discussed wind tunnel will reach fully developed turbulent flow. Nevertheless, even if it happens, the location of the turbulent flow separation is roughly at $\sim 130 \mathrm{deg}$. This means in both cases separation locations will be sufficiently far upstream from the position where there is the neck of the flask. The neck is likely to be inside the wake where the flowfield condition are not suitable anyway for detailed investigations.

\section{Governing dimensionless parameters}

While the flow of heated air past the test object is governed by the Reynolds number, eq. (2), the processes of heat transfer are expected to depend upon the product of two dimensionless parameters, Fourier number Fo, defined in Fig. 14, and the Stefan number Sf, defined in Fig. 15.

$$
\mathrm{t} \sim \text { Fo.Sf }
$$

Fo characterises time dependent, unsteady processes without phase change, in which it represents the nondimensionalised time $\mathbf{t}$. The multiplication by Sf results in the expressions which characterise the processes involving the latent heat.

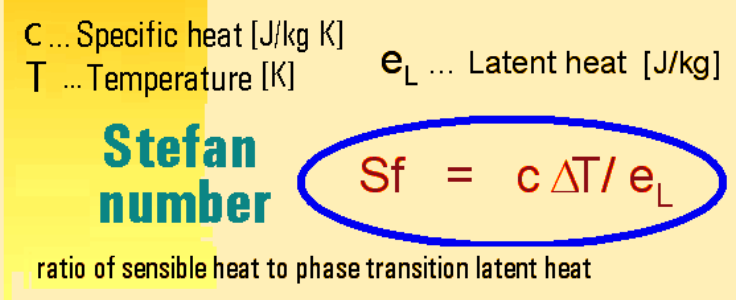

Fig. 15. Stefan number $\mathrm{Sf}$ is expected to be the governing dimensionless parameter of prime importance in processing the experimental data obtained for the melting and solidification studies in the capsule models.

It is difficult to estimate to what degree the heat propagation inside the encapsulation will be limited only to heat transport by conduction. It cannot be excluded that at the larger geometric scales there may appear effects of internal natural convection. In that case a certain role may be also played by Grasshoff number, defined

$$
\mathrm{Gr}=\left(\mathrm{g} \boldsymbol{\beta}_{\mathrm{v}}\left(\mathrm{T}-\mathrm{T}_{\mathrm{m}}\right) \mathrm{d}^{3}\right) / \boldsymbol{v}^{2}
$$

- where $\mathrm{g}=9.81 \mathrm{~m} \mathrm{~s}^{-2}$ is gravitational acceleration, $\beta_{\mathrm{V}}[-]$ is coefficient of volumetric expansion of the sample in liquid state, $\mathrm{T}[\mathrm{K}]$ is the temperature inside the sample in solid state, and the rest are parameters that were already introduced above.

It may be expected that plotting the time dependence of the solidification processes after conversion into the dimensionless variables will be similar to what is presented in Fig. 16. On the horizontal co-ordinate is the dimensionless time eq. (3) while on the vertical coordinate is plotted the relative percentage of the volume of tested material that remains during solidification in molten state (related to the initial $100 \%$ volume). Typical heat transfer processes, because of the character of the governing Fourier equation, usually exhibit exponential dependence. In the semi-logarithmic co-ordinates used to plot the data in Fig. 16, such dependence would be fitted by a straight line. This is what is likely to be found in the settled regime at a longer time after the start of cooling. Of as yet not well understood character will be quite probably the initial 


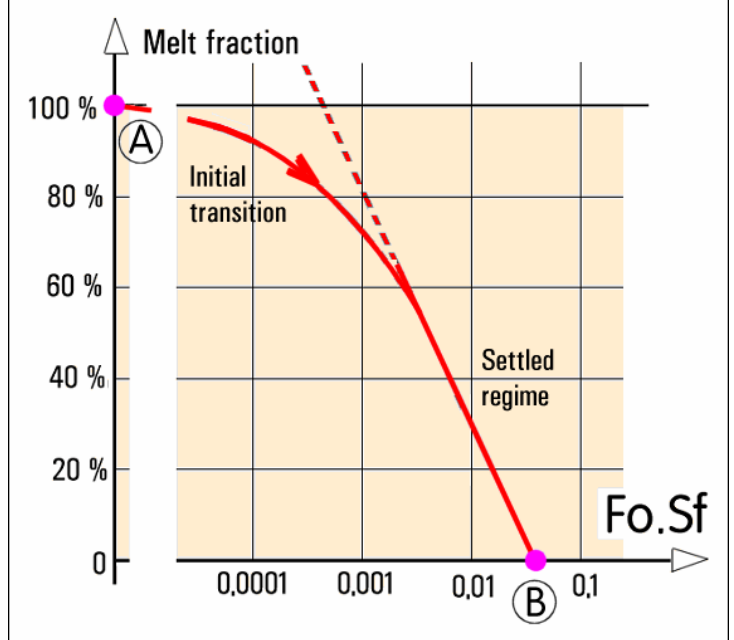

Fig. 16. Expected character of measured data on the solidification inside the spherical capsule model after their conversion into dimensionless dependence in semi-logarithmic co-ordinates . Essential part of the plotted curve is exponential (and therefore a shown here as straight line). Very little is so far known about the very short time of initial transition.

regime of small Fo.Sf values. To answer this question, it seems to be necessary to concentrate the attention in the planned experiments on the initial regime and its transition into the exponential dependence - i.e. in the time range largely so far out of interest.

\section{Wind tunnel layout}

The experiments aimed at answering the above mentioned questions (and some other ones related to it) are to be performed in the configuration as shown in Fig. 12 - where the initially hot air flow past the tested spherical shell, securing a complete melting, is to be suddenly and very rapidly switched to a cold air flow. The suddenness is important for obtaining data in the initial transition regime seen in Fig. 16. This is a requirement that was so far never posed in wind tunnel designs.

The requirements placed on general tunnel configuration included the starting point heating to (and, if desirable, also above) approximately $\mathrm{T} \sim 60{ }^{\circ} \mathrm{C}$ followed by the sudden switching to cold air flow. If such temperature changes were made in a direct manner it would be necessary to apply very high heating an cooling power input. The obvious problem is the thermal lag: heated components of the tunnel are likely to keep considerable stored heat after the switching. Considering these aspects - and also the availability of reasonable power input in the laboratory - it became obvious that the molten-material regime necessitates re-circulating the hot air flow. Thus the wind tunnel design ideas had to concentrate on the closed-circuit tunnel layouts. The vertical flow in the test section as presented in Fig. $12-$ which, of course, is a part of the recirculatory airflow necessitated orientation of the circuit in the vertical plane. The resultant configuration is as presented in Fig. 17. To avoid a large structure that would be necessary with

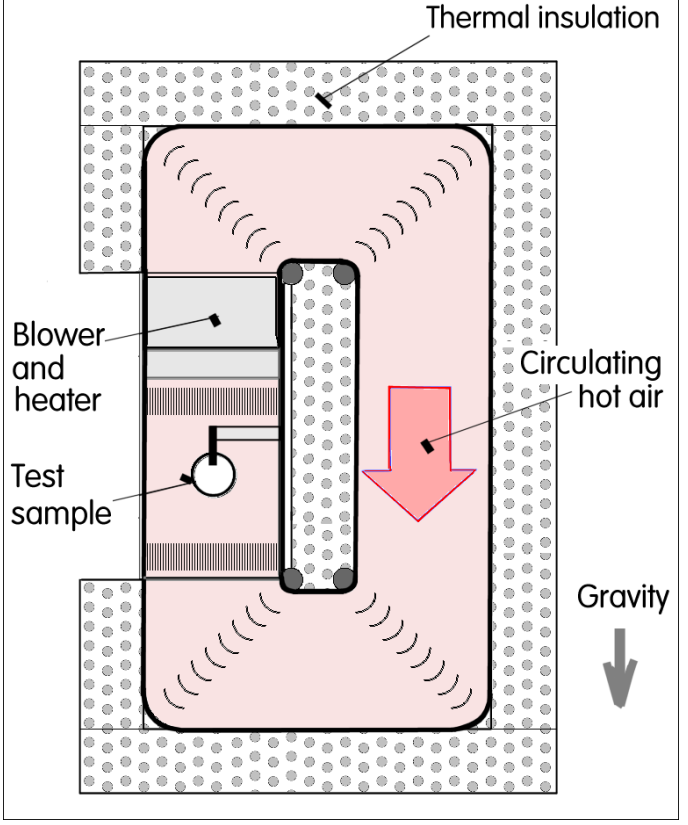

Fig. 17. Layout of the currently built wind tunnel for the latent heat tests. Hot air flow circulates in a vertically oriented loop. In the "up" arm at left is the test section and above it the combined unit for driving and heating the flow.

simple large turning-radius elbows (to avoid flow separations from the walls) in the return part of the tunnel, the design was focused on the historic approach of Prandtl's Göttingen tunnel. The elbows chosen were of sudden 90 deg turning type the flow separation eliminated by a rather dense turning blades. As shown in

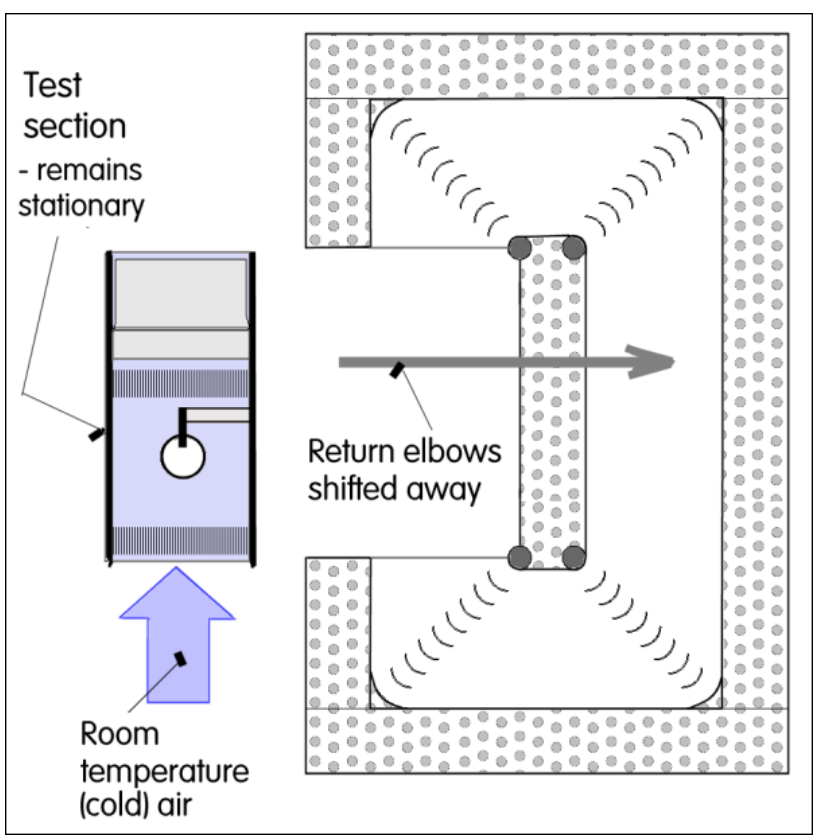

Fig. 18. Operation of the wind tunnel in the sample solidifying test. At the start of the cooling run the whole return-flow part of the tunnel is shifted away while the test section remains in place and is fed by cold air from the room. The stationary position of the test section is necessary for retaining properly focused cameras and lights (also not shifted). 


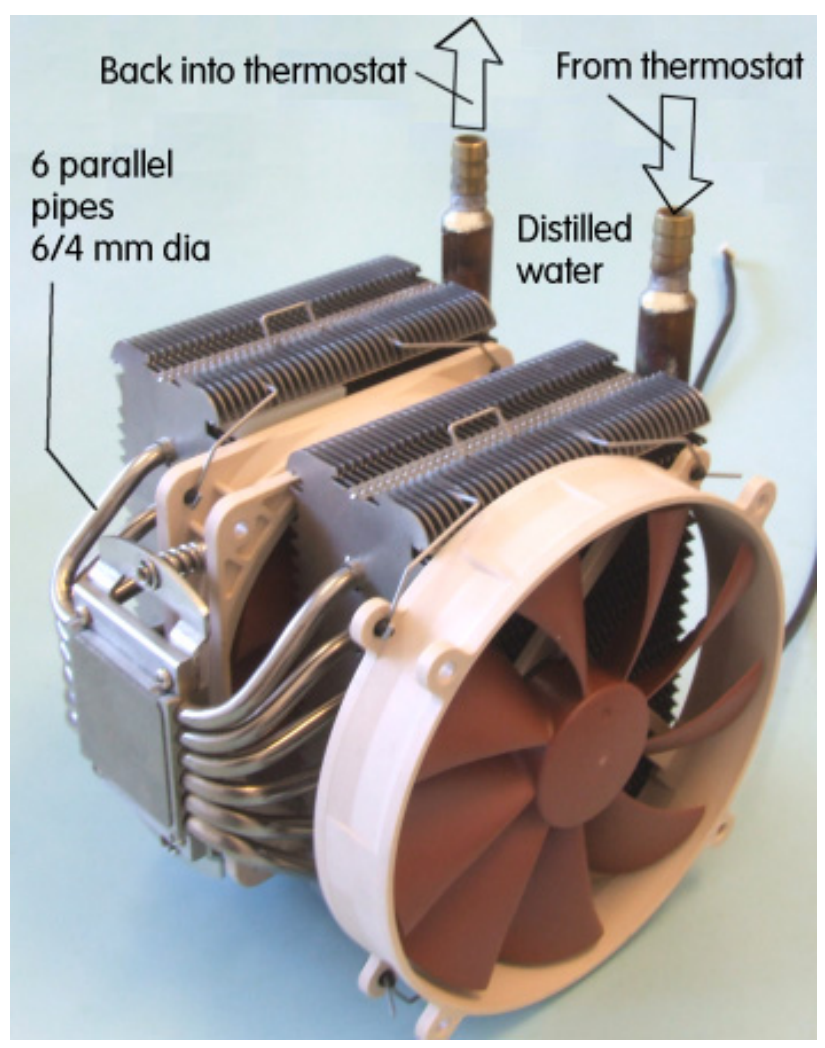

Fig. 19. Photograph of the heating and driving unit. Note the two series of the sheet-metal fins and the Ni coated copper pipes supplying them with heat from the thermostat.

Fig. 17, each of the four elbows are provided with a row of 8 turning blades. For further improvement of the velocity profile inside the test section, below and above the test section the whole tunnel cross section is filled with shortened packed drinking straws.

The blower unit inevitably destroys somewhat the character of the flow and to minimise its effect it is positioned above the test section. Swirl flow is suppressed by the heat-transfer find in the unit, as described below.

Heating of the air flow is secured by heat exchanger forming a part of the unit together with the two fans. Heat is transferred into the air through a grid of rather densely packed thin metal fins. Transfer into the fins is by tubes connected into a thermostat operating with recirculating distilled water. Thermostat allows precise adjustment of the distilled water temperature. The thermostat is positioned immediately below the wind tunnel.

For the sudden switching from the hot air flow above the melting temperature into a cold air flow regime, the solution was found in placing the whole return-flow part of the wind tunnel on rails. As shown in Fig. 18, the return duct may be suddenly shifted away so that the blower starts immediately delivering into the test section the cool room temperature air.

\section{Driving and heating unit}

The wind tunnel design discussed above was based on the idea of commercial availability the suitable unit generating the hot air flow. In this unit the air flow is to be driven and simultaneously heated to and above the paraffin melting temperature $\mathrm{T}_{\mathrm{m}}$. The suitable unit, originally intended for a different task, was selected in manufacturers' catalogue on the Internet. It is presented in the photograph Fig. 19. It was designed for use in cooling a computer CPU.- and here it is adapted for a slightly different role. It is specified as Noctua NH-D14 6-heatpipe with dual NF-A15 140 fans - the last number 140 indicates the diameter of the fans in millimetres. In its original use unit is provided with the baseplate (seen at left in Fig. 19) for fixing to this location the CPU. The heat from the baseplate was to be removed by $12(=2 \mathrm{x}$ 6 ) heat pipes and transferred to two series-connected grids of thin aluminium fins exposed to the air flow.

For the use in the developed wind tunnel the internal components of the heat pipes were removed and the ends of the pipes were provided with two manifolds. Each manifold was tin-soldered to six pipes and contained a ferrule to serve as inlet or (on the opposite side) an outlet of heated distilled water from the thermostat. The baseplate for the CPU is retained, though it now does not serve for any particular purpose. The positioning of the unit inside the test section is shown in Fig. 20. Each of the two fans, operating in series, has an airscrew of 180 $\mathrm{mm}$ diameter with 9 blades. Driving power for each airscrew is $0.6 \mathrm{~W}$.

The unit is to be positioned into the $140 \mathrm{~mm} \times 140$ $\mathrm{mm}$ test section of the tunnel as presented in Fig. 12. The rotational speed of the fans is adjustable in the PWM (pulse width mode) regime. In the manufacturer's catalogue is stated that the fans are fully controllable from 600 RPM minimum speed to maximum 1300 RPM. The flow rate at this maximum rotation speed is

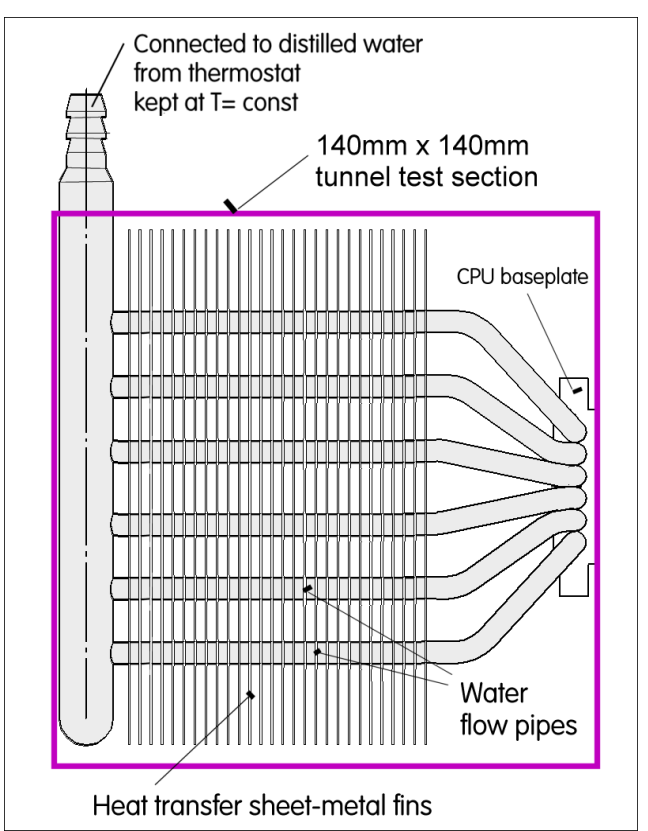

Fig. 20. Layout of the dual air heater. Water from the thermostat is distributed in each part of the heater by six pipes to a series of parallel thin aluminium fins. The original baseplate for CPU was retained despite its now having no useful role. 


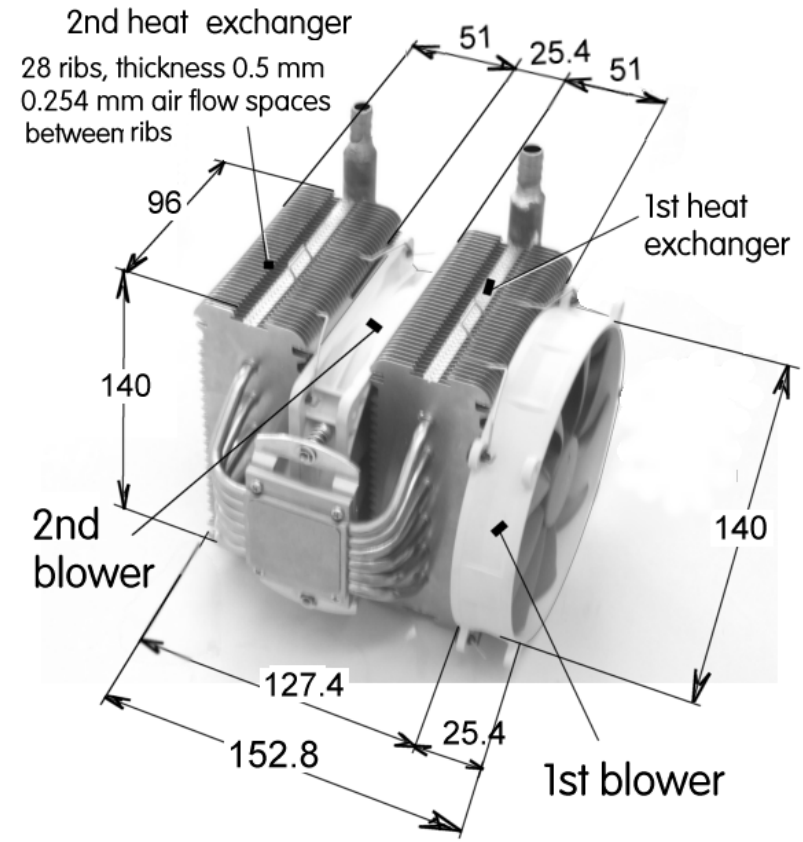

Fig. 21. The unit used to drive the circulating heated air in the wind tunnel by the two blowers - and also maintaining its temperature by means of the two-component heat exchangers. Liquid with precisely adjusted temperature is supplied from an external thermostat unit.

64.9 CFM (cubic feet per minute) which represents $0.0332 \mathrm{~m}^{3} / \mathrm{s}$. The cross-section area of the empty wind tunnel test section is $0.0196 \mathrm{~m}^{2}$. If the wind tunnel is run with the largest, $\mathrm{d}=64 \mathrm{~mm}$ diameter spherical shell (cf. Figs. 12 and 13), then the available cross-sectional area for the air flow is reduced to $0.0164 \mathrm{~m}^{2}$. Neglecting the losses in the return duct of the wind tunnel, at the maximum rotational speed of the fans the airflow velocity is approximately $2.0 \mathrm{~m} / \mathrm{s}$. With cold air of viscosity 15.7 $10^{-6} \mathrm{~m}^{2} / \mathrm{s}$ the Reynolds number of the flow past the tested sample under these conditions is roughly $\operatorname{Re} \sim 8.2510^{3}$. When run with $60{ }^{\circ} \mathrm{C}$ hot air of viscosity $18.810^{-6} \mathrm{~m}^{2} / \mathrm{s}$, the Reynolds number will be approximately $7.110^{3}$.

The lowest Reynolds number of the hot air flow obtained by continuous electronic PWM control, i.e. when run at the value 600 RPM minimum fan speed indicated in the manufacturer's catalogue with the smallest, $d=35 \mathrm{~mm}$ diameter spherical shell (cf. Fig. 13), the same calculation shows Reynolds number $\operatorname{Re}=1 \cdot 10^{3}$. It is, of course, easily possible to switch off one of the two fans to obtain approximately $\operatorname{Re}=0.510^{3}-$ and even much lower values may be obtained by mechanical area blockage in the return flow.

\section{Wind tunnel body and progress report}

At the time of writing this paper (June 2017) the wind tunnel is still being built. The associated photographs Figs. 22 and 26 show it in its not yet finished state.

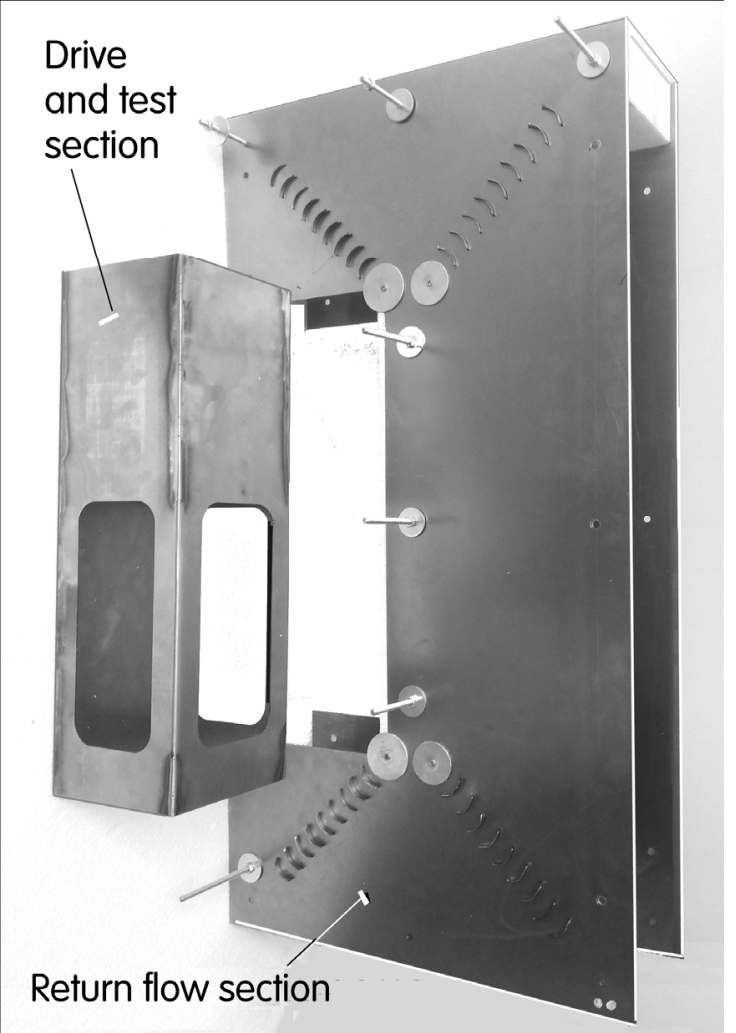

Fig. 22. Photograph of the two main components of the wind tunnel in construction. Both are made of steel sheets as the shape-carrying structure - at left is the test section with the large rectangular windows, at right is the flow-return part. The latter will be covered with thermal insulation cladding of thermal insulation thick polystyrene foam plates.

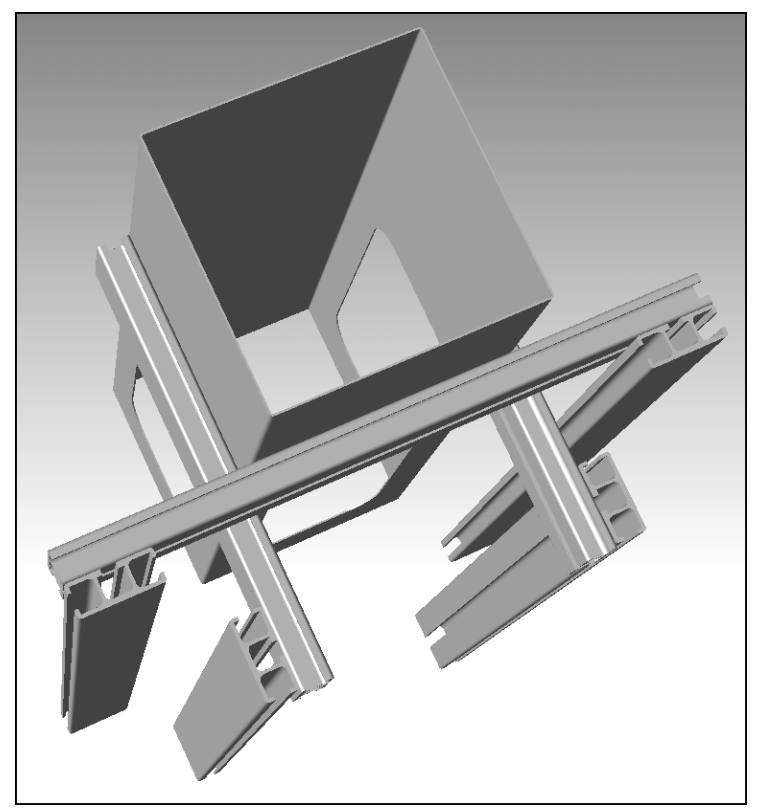

Fig. 23. Rather complicated design of fixating the test section (while the rest of the tunnels is translatable on rails) was necessitated by the request all three large access windows being simultaneously accessible for cameras. The components of the fixation structure are standard aluminium-alloy profile rods, commercially available. Triangular holders as well as the screws are here not shown. 


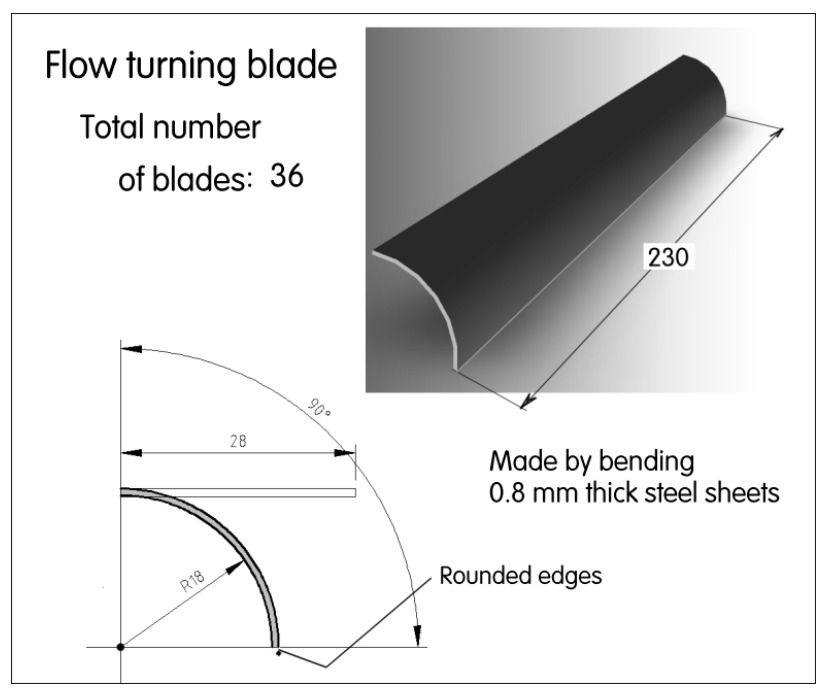

Fig. 24. Simple turning blades made by bending a $0.8 \mathrm{~mm}$ sheet metal rectangles, of constant thickness with rounded leading edge.

There are two basic parts: at left side of both photographs it is the test section housing the scaled-up model under test as well as the unit from Fig. 21. On the right-hand side in these photographs is the body of the return-flow tunnel part. It is on wheels running in rails so that this whole large part may be shifted away from the test section (at the time of taking the photographs the wheels and rails were still missing).

The test section is made from steel sheets bent and welded into a rectangular object. Well visible feature are the large rectangular holes that serve as access and observation windows. One is in the front wall and the other two are two on both sides. The holes are going to be covered by polymethylmethacrylate (transparent plexiglass) plates - held on screws so that they be removed for access to the test model. The front window may be replaced by thermocamera observation port is now already provided in the wind-tunnel test section as an alternative to one of the plexiglass windows. It is a round, $30 \mathrm{~mm}$ dia disk of $\mathrm{ZnSe}$, supplied by Eksma

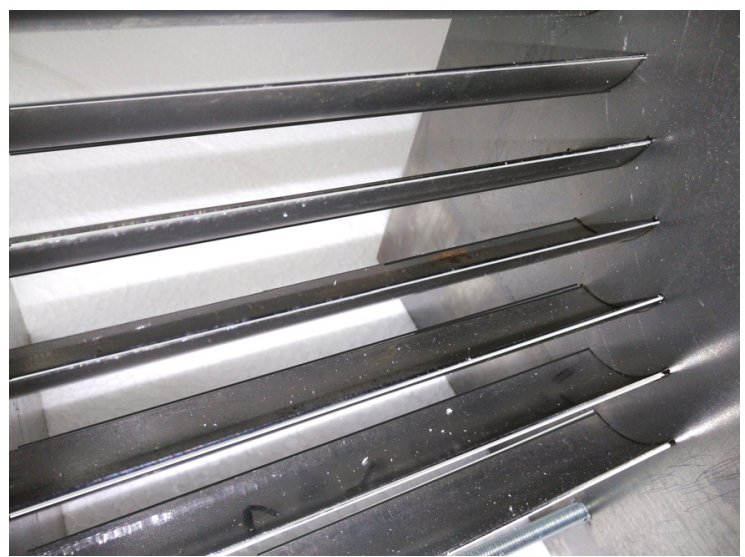

Fig. 25. The close-up photograph of the flow-turning blades (their detail is in Fig. 24) already fixed to their position in the return-flow duct.

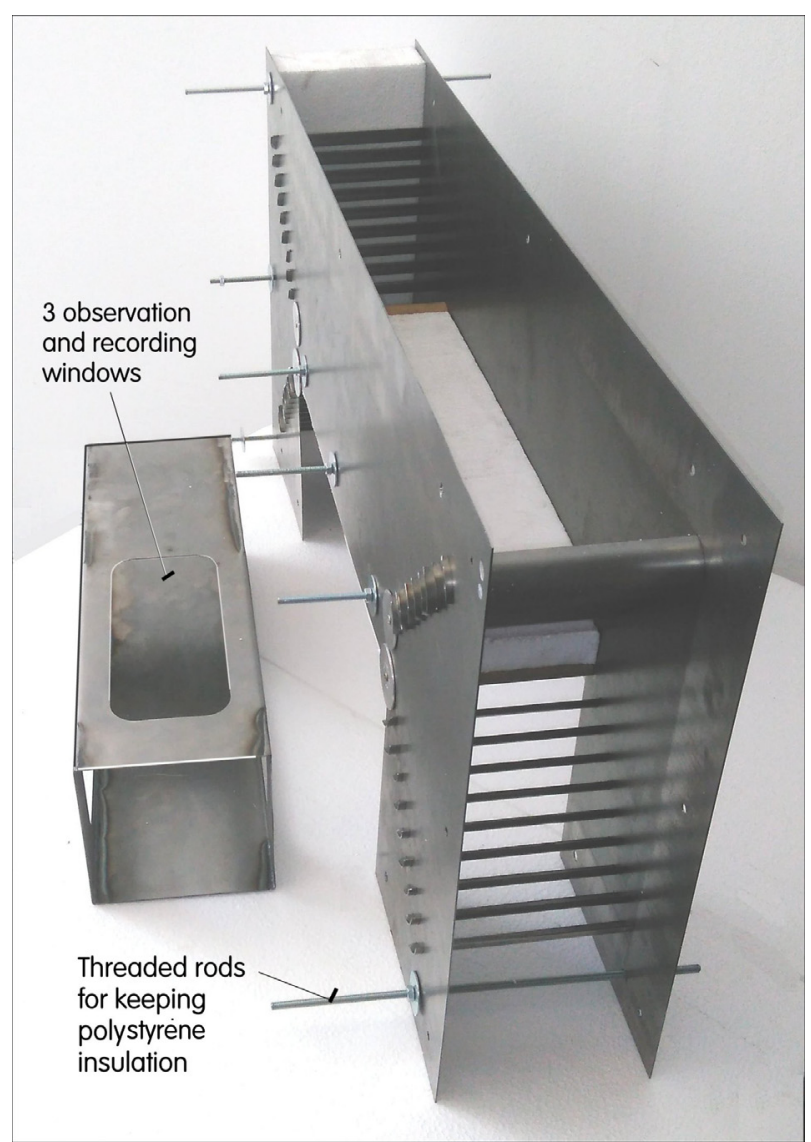

Fig. 26. Photograph of the two components of the tunnel. The polystyrene foam thermal insulation cladding will be held on the threaded rods seen in this photograph. The cladding will leave for the air flow the flowpath as seen in Fig. 27.

Optics in Lithuania. It is necessary because plexiglass blocks infrared light of wavelengths longer than 2800 $\mathrm{nm}$ [17]. The test section is fixed to the stand by the structure shown in Fig. 23. Somewhat complex design is due to the request of no obstruction of any of three windows - which are quite wide so that the noobstruction requirement resulted in placing the vertical members of the structure outside the test section corners, as is visible in the picture Fig. 23.

On the right-hand aside of the photographs Figs. 22 and 26 is the return duct part of the wind tunnel. Its basic components are the quite large sheet-metal plates - the left and right ones. Both are held together by the total of 32 corner flow-turning blades with the whole welded together. On the bottom part of this whole unit (not seen the photographs here) are standard commercially available metal rails - and wheels running in them. Workshop drawing of the detail of flow-turning blades is presented in Fig. 24. The blades are very simply made by bending from a $0.8 \mathrm{~mm}$ thick steel sheet rectangles - and are therefore of the everywhere constant thickness. Only the leading edges are rounded, as may be recognised from the close-up photograph of the blades in their positions in Fig. 25. As was mentioned above, the blades, apart from their flow-turning role, also hold the two steel sheets (as seen in Fig. 26) together at the $140 \mathrm{~mm}$ mutual separation distance. 


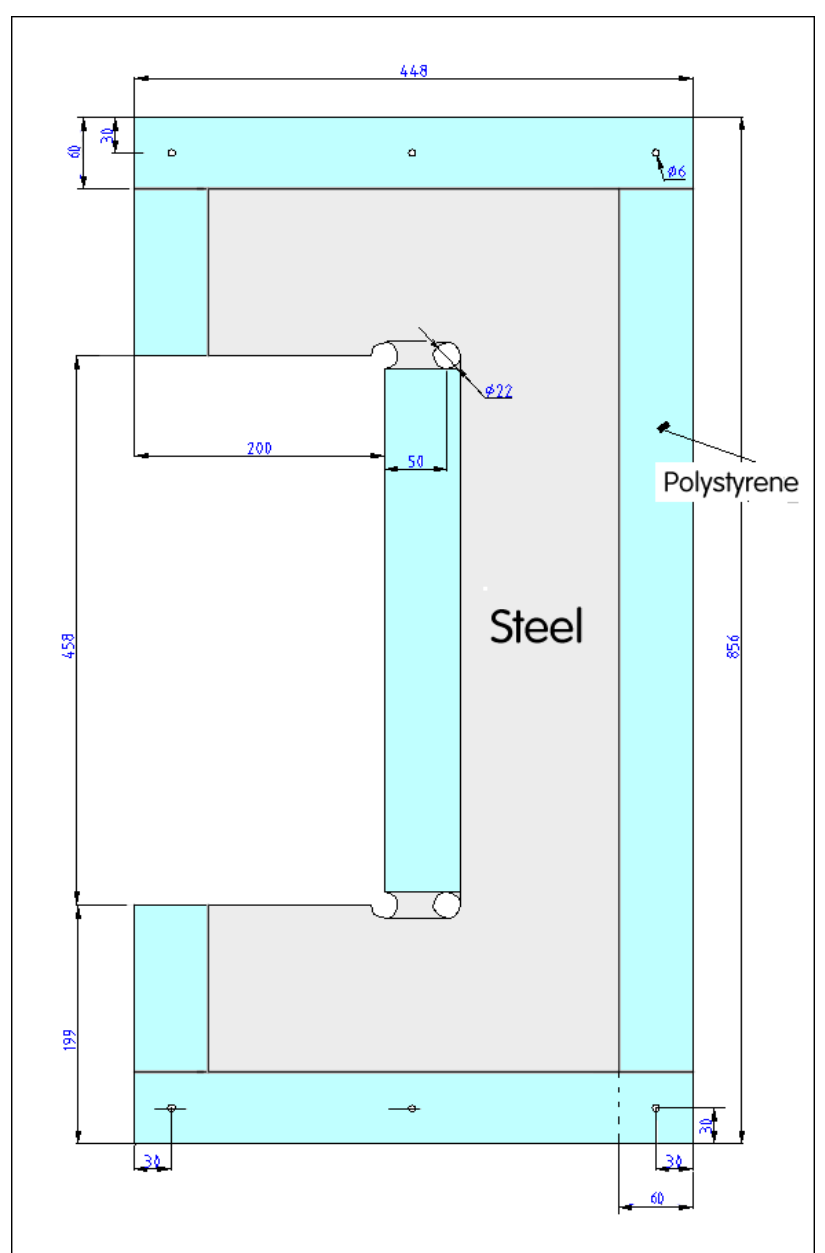

Fig. 27. Position of the $60 \mathrm{~mm}$ thick polystyrene block inside the sheet-metal walls. Available for the airflow is the grey colour area.

Still missing in the attached photographs is the covering by $60 \mathrm{~mm}$ thick thermal protection polystyrene foam plates. They will be held in position on the outer side of the sheet-metal plates using the threaded rods particularly well seen as projecting from the steel plate in Fig. 26. Made of polystyrene foam plates, $60 \mathrm{~mm}$ thick and $140 \mathrm{~mm}$ wide, will be also the components placed between both steel plates in the configuration presented in Fig. 26. They will delimit the return flowpath of the hot air. Since the melting and solidification tests are planned to be performed with paraffin melted at temperatures only slightly above the temperature of human body, there is no problem foreseen with the direct contact of the hot air and the plates of polystyrene which melts in the temperature range $210-249^{\circ} \mathrm{C}$ (depending on the precise sort).

\section{Conclusions}

This paper provides essential information about progress in the grant project started in January 2017. It aim is obtaining basic information and its use in development of thermal energy storage system using the energy stored in the form of latent heat - i.e. by using it to melting a solid object. The advantage of this approach is the possibility of storing quite large amount of heat in a relatively small melted body, without operating with high temperatures. The first part of the paper provides collected information and some useful data about the phase-change materials. Described in the second part of the paper is a very special small wind tunnel. It should serve for studies of melting and solidifying paraffins, encapsulated in a scaled up spherical shell. The extraordinary feature of the tunnel, which is now built, is the capability of performing a sudden step change of the temperature. .

\section{Acknowledgements}

Author was financially supported by the Czech Republic Grant Agency research grant Nr. 17-08218S and also by institutional support RVO: 61388998.

\section{References}

1. J. W. Gibbs, Scientific Papers, Dover, New York, 1961

2. H. Chisholm, "Black Joseph", Encyclopaedia Britannica 4, Cambridge Univ. Press, 1911

3. K. Karthik et al., Journal of Textile and Apparel, Technology and Management 7, 3, 2012

4. G. Haoshan, L. Jing, Journal of Heat Transfer 135, 054503, 2013

5 N. Leoni, C.H. Amon, C. H., ASME HTD 343, pp. 49-56, 1997

6. I. Harary et al., International Journal of Thermodynamics $\mathbf{1 9}$, $36-41,2016$

7 K.G. Steenbergen, Modelling the Melting of Gallium Clusters, PhD Thesis, Univ. of Wellington, New Zealand, 2013

8. Ł. Wierbicki, Journal of Achievements in Materials and Manufacturing Engineering, p. 26, 2014

9. Anonymous, "Praffin hydrocarbon", Encyclopaedia Britannica, 2015

10. Anonymous, "Alkyl Groups", IUPAC, Compendium of Chemical technology, $2^{\text {nd }}$. ed., 2015

11. H. Plessen, "Sodium Sulfates" in Ullmann's Encyclopedia of Industrial Chemistry Wiley-VCH, 2000

12. E. Assis, G. Ziskind, Letan R., Journal of Heat Transfer 131, 024502-1, 2009

13. A. Felix Regin, S.C. Solanki, J.S. Saini, Renewable and Sustainable Energy Reviews 12, 2438-2458, 2008

14. F.A.P.S. Scacchetti, E. Pinto, G.M.B. Soares, Prograss in Oreganic Coatings, 107, 64-74, 2017

15. S. M. Zhang, T. Noll, D. Houtman, Thermal and Thermomechanical Phenomena in Electronic Systems, ITHERM 2002. Conf. San Diego, 2002

16. W.G.Wolfer, B. Oudot, N. Baclet, Journal of Nuclear Materials, 359, 185-191, 2006

17. N. L. Chiromawa, K. Ibrahim, Internat. Conf. of Global Network for Innovative Technology IGNITE 2014 